\title{
Antidepressant Effects of Soyo-san on Immobilization Stress in Ovariectomized Female Rats
}

\author{
Jin Kyung OH, ${ }^{a}$ Yoon-Sang Kim, ${ }^{b}$ Hyun-Jung PARK, ${ }^{c}$ Eun-Mee Lim, ${ }^{b}$ Kwang-Ho PyUN, ${ }^{c}$ and \\ Insop SHIM*,c \\ ${ }^{a}$ Department of Oriental Medical Science, Graduate School of East-West Medical Science, Kyung Hee University; 1 \\ Seochon-ri, Kiheung-eup, Youngin-shi, Kyungki-do 449-701, South Korea: ${ }^{b}$ Department of Oriental Gynecology, Oriental \\ Hospital, College of Oriental Medicine, Kyung-won University; 461-701, South Korea: and ${ }^{c}$ Department of Integrative \\ Medicine, College of Medicine, The Catholic University of Korea; 137-701, South Korea.
}

Received October 18, 2006; accepted April 20, 2007

Soyo-san is a traditional oriental medicinal formula, a mixture of 9 crude drugs, and it has been clinically used for treating mild depressive disorders. The purpose of the study was to examine the effect of Soyo-san on repeated stress-induced alterations of learning and memory on a Morris water maze (MWM) task and also the anxiety-related behavior on the elevated pulse maze (EPM) in ovariectomized female rats. We assessed the changes in the reactivity of the cholinergic system by measuring the immunoreactive neurons of choline acetyltransferase (ChAT) and reactivity of acetylcholinesterase (AChE) in the hippocampus, and the serum levels of corticosterone were assessed after behavioral testing. The female rats were randomly divided into three groups: the nonoperated and nonstressed group (normal), the ovariectomized and stressed group (control), and the ovariectomized, stressed and Soyo-san treated group (SOY). The rats were exposed to immobilization stress (IMO) for $14 \mathrm{~d}(2 \mathrm{~h} / \mathrm{d})$, and Soyo-san $(400 \mathrm{mg} / \mathrm{kg}$, i.p.) was administered $30 \mathrm{~min}$ before IMO stress. Treatments with SOY caused significant reversals of the stress-induced deficits in learning and memory on a spatial memory task, and it also produced an anxiolytic-like effect on the EPM, and increased the ChAT and AChE reactivities $(p<0.05$, respectively). The serum level of corticosterone in the SOY group was significantly lower than that in the control group $(\boldsymbol{p}<\mathbf{0 . 0 5})$. These results suggest that Soyo-san might prove to be an effective antidepressant agent.

Key words Soyo-san; depression; ovariectomy; Morris water maze; elevated pulse maze; choline acetyltransferase

Depressive disorders are among the most frequently occurring psychiatric diseases, and are prevalent in at least $15 \%$ of the population. However, the prevalence of depressive disorders is twice as high in women as in men. ${ }^{1)}$ The higher incidence and severity of depression is associated with the presence or absence of ovarian hormones. ${ }^{2)}$ The ovarian hormones related to the menopause-induced depression influence the hippocampal anatomy and physiology; thus, they affect behavior in adult female rats. ${ }^{3-5)}$ The hippocampus plays an important role in the negative feedback mechanism of the limbic-hypothalamic-pituitary adrenal (LHPA) axis. It expresses high levels of adrenal steroid receptors and is especially susceptible to damage as a result of stress. ${ }^{6,7)}$ In the hippocampus, stress hormones induce regression of the dendritic processes and cell death, inhibit neurogenesis, and impair the ability of neurons to survive noxious stimuli such as seizure, hypoxia-ischemia, metabolic poisons, hypoglycemia and oxygen radical generators. ${ }^{8)}$ The damage caused by a heightened stress response resulting from dysregulation of the LHPA axis is hypothesized to play a role in the reduced hippocampal volume often associated with major depression. ${ }^{9,10)}$ Several neurotransmitter systems implicated in the regulation of the HPA axis have been hypothesized to be dysfunctional in major depression, including acetylcholine (ACh), norepnephrine, serotonin and dopamine. ${ }^{11)}$ The central cholinergic neurons are of putative relevance to major depression. $^{12)}$

Soyo-san consists of 9 kinds of medicinal herbs: Paeoliae Radix Alba, Atractylodis Macrocephalae Rhizoma, Angelicae Gigantis Radix, Poria, Liriopis Tuber, Bupleuri Radix, Menthae Herba, Glycyrrhizae Radix and Zingiberis Rhizoma
Recens. Soyo-san is a traditional oriental herbal medicine, and it has been used in the treatment of mental stress, hypochondriac distensive pain, hysteria and manic-depressive illness. The various findings of recent preclinical studies have supported the therapeutic value of Soyo-san in a clinical setting. ${ }^{13,14)}$ In laboratory studies of animals, Soyo-san has also been shown to have antidepressive-like effects when using the tail suspension and forced swimming tests. ${ }^{15,16)}$ In animal models, it was suggested that exposure to uncontrollable and stressful life events led to a feeling of 'loss of control' which ultimately led to depression. In this 'learned helplessness' response, animals exposed to stressors have impaired performance in learning and memory. ${ }^{17}$ )

The aim of the present study was to explore the behavioral and the neurobiological effects of Soyo-san on ovariectomized female rats and to form a basis for clinical treatment. Soya-san's antidepressant effect was tested via a Morris water maze (MWM) and an elevated pulse maze (EPM), and we measured the serum corticosterone and immunohistochemical changes of acetylcholine in the brain.

\section{MATERIALS AND METHODS}

Preparation of Drug Soyo-san was prepared by mixing the 9 crude drugs in the same ratio (Table 1). It was provided by the department of Oriental Medicine, Graduate School of Kyungwon University. This herbal mixture was soaked in distilled water (10 fold the volume of the mixture). After boiling at $100^{\circ} \mathrm{C}$, the decoction was filtered through filter paper. The water extract was concentrated by heating the mix at $60^{\circ} \mathrm{C}$ for $2 \mathrm{~h}$ before the extract was dried to a powder 
Table 1. Composition of Soyo-san

\begin{tabular}{ll}
\hline \multicolumn{1}{c}{ Components } & $(\mathrm{g})$ \\
\hline \hline Paeoliae Radix Alba & 4 \\
Atractylodis Macrocephalae Rhizoma & 4 \\
Angelicae Gigantis Radix & 4 \\
Poria & 4 \\
Liriopis Tuber & 4 \\
Bupleuri Radix & 4 \\
Menthae Herba & 2 \\
Glycyrrhizae Radix & 2 \\
Zingiberis Rhizoma Recens & 6 \\
Total & 34 \\
\hline
\end{tabular}

under anaerobic conditions for $4-5 \mathrm{~d}$ (yield of the extract was $20 \%$ ). The extract was then freeze-dried and stored at $-20{ }^{\circ} \mathrm{C}$ until use.

Animals and Experimental Design Female Sprague Dawley rats at the age of 8 weeks (Samtaco, Inc. Korea) were used for the study. The rats were housed under a controlled temperature $\left(22-24^{\circ} \mathrm{C}\right)$ with a $12 \mathrm{~h}$ light/dark cycle. The lights were on from 8:00 to 20:00. Food and water were made available ad libitum. They were allowed at least 1 week to adapt to their environment before the experiments.

The female rats were randomly divided into three groups ( $n=6$ per group): the nonoperated and nonstressed group (normal), the ovariectomized and stressed group (control), and the ovariectomized, stressed and Soyo-san treated group (SOY).

Using aseptic conditions, bilateral ovariectomy was performed under general anesthesia with pentobarbital sodium $(50 \mathrm{mg} / \mathrm{kg}$, i.p.). After postoperative recovery for $7 \mathrm{~d}$, the ovariectomized rats were stressed daily. The stress was produced by forcing the animals into an immobilizer device (a disposable rodent restraint cone, Havard Instrument, U.S.A.) for $2 \mathrm{~h}(10: 00-12: 00$ a.m. $)$ for $14 \mathrm{~d}$.

From the 8th day after the first immobilization, the SOY group was daily treated with the Soyo-san extract $(400 \mathrm{mg} /$ $\mathrm{kg}$, i.p.) for 2 weeks, and other groups were given sterile saline. Immobilization began $30 \mathrm{~min}$ after the treatments.

Morris Water Maze Test On the first day of injection to induce immobilized stress, all the animals started training on a MWM task in a swimming pool $(1.8 \mathrm{~m}$ diameter and $0.5 \mathrm{~m}$ high, filled with milky water at a temperature in the $22 \pm 2{ }^{\circ} \mathrm{C}$ range) for $7 \mathrm{~d}$. A $12 \mathrm{~cm}$ diameter round platform was hidden in a constant location (the quadrant center) within the pool with its top surface submerged $1.5 \mathrm{~cm}$ below the water level. The rats were trained to locate the hidden island in four trials per day for $6 \mathrm{~d}$. After the $6 \mathrm{~d}$, they were started in the quadrant opposite to the target and were forced to swim for $60 \mathrm{~s}$ in the pool without a platform. The spatial memory of the rats was assessed as the latency time. The proportion of time the rat spent searching for the platform in the training quadrant, i.e., the previous location of the platform, was recorded and used as a measure of memory retention. A video camera was mounted on the ceiling above the pool and was connected to a video-recorder and tracking device (S-MART; Pan-Lab, Barcelona, Spain), which permitted on-line and off-line automated tracking of the path taken by the animal.

Elevated Plus Maze Test EPM tests were performed on the 8th day after $7 \mathrm{~d}$ of MWM testing. After treatment, the control and SOY groups underwent immobilized stress for
5 min before the EPM test. The construction and testing procedure of the EPM were based on a method described by Pellow et $a l .{ }^{18)}$ The frequency of entries into the open arms and the closed arms of the maze and the time spent on the respective arms were recorded for a $5 \mathrm{~min}$ period.

Histochemistry Animals were anesthetized with sodium pentobarbital $(100 \mathrm{mg} / \mathrm{kg}$, i.p.) and then perfused transcardially with $100 \mathrm{ml}$ of saline followed by $500 \mathrm{ml}$ of a $4 \%$ solution of formaldehyde prepared in phosphate buffer. The brains were then removed, postfixed in the same fixative for two to three hours at $4{ }^{\circ} \mathrm{C}$ and then placed overnight at $4{ }^{\circ} \mathrm{C}$ in PBS containing 20\% sucrose. The following day, the brain was cut into coronal sections that were sliced to $30 \mu \mathrm{m}$-thicknesses.

Sections were processed for choline acetyltransferase (ChAT) immunoreactivity using sheep anti-ChAT polyclonal antibody (Chemicon international, Temecula, CA, U.S.A.). The primary antibody was prepared at a dilution of $2000 \times$ in $0.3 \%$ PBST, $2 \%$ normal rabbit serum and $0.001 \%$ kehole limpit hemocyanin (Sigma, U.S.A.). The sections were incubated in the primary antiserum for $72 \mathrm{~h}$ at $4{ }^{\circ} \mathrm{C}$. Following rinsing in PBST, the sections were incubated for $2 \mathrm{~h}$ at room temperature in biotinylated rabbit anti-sheep secondary antibody (Vector Laboratories, Burlingame, CA, U.S.A.) that was diluted $200 \times$ in PBST containing $2 \%$ normal rabbit serum. After three more rinses in PBST, the sections were placed in Vectastain Elite ABC reagent (Vector Laboratories, Burlingame, CA, U.S.A.) for $2 \mathrm{~h}$ at room temperature. Following a further rinsing in PBS, the tissue was developed using diaminobenzadine (Sigma, U.S.A.) as the chromogen.

For the acetylcholinesterase (AChE) histochemistry, the sections were incubated for $1-2 \mathrm{~h}$ at room temperature in $0.1 \mathrm{M}$ sodium hydrogen phosphate buffer $\left(325 \mathrm{ml}, \mathrm{NaH}_{2} \mathrm{PO}_{4}\right.$. $\left.\mathrm{H}_{2} \mathrm{O}, \mathrm{pH} 6.0\right)$ that contained acetylcholine iodide $(250 \mathrm{mg})$, to which $0.1 \mathrm{~m}$ sodium citrate $(25 \mathrm{ml}), 30 \mathrm{~mm}$ copper sulfate $(50 \mathrm{ml}), 50 \mathrm{~mm}$ potassium ferricyanide $(50 \mathrm{ml})$ and distilled water $(50 \mathrm{ml})$ were added.

The number of target neurons was counted at $400 \times$ magnification using an optical microscope (Olympus $\mathrm{BH} 2$, U.S.A.) with a rectangular grid that measured $200 \times 200 \mu \mathrm{m}$. For counting neurons, the grid was placed around the areas according to the Paxinos and Watson atlas. ${ }^{19)}$ The density of the AChE-neurons was measured using the Scion image program (Scion Corp., MD, U.S.A.).

Corticosterone Immunoassay After the behavioral test, the animals were deprived of food for $24 \mathrm{~h}$ and then were anesthetized with sodium pentobarbital $(100 \mathrm{mg} / \mathrm{kg}$, i.p.). Blood was collected via the abdominal aorta. The isolated serum was stored at $-70^{\circ} \mathrm{C}$ until assay. Corticosterone was measured by a competitive enzyme immunoassay using a rabbit polyclonal corticosterone antibody (Octeia Corticosterone: Alpco Diagnostics, American Laboratory Products, Windham, NH, U.S.A.)

Data Analysis Statistical comparisons were done for the behavioral and histochemical studies using repeated measures of ANOVA and the one-way ANOVA, respectively, and LSD post hoc comparisons were also done. All of the results were presented as means \pm S.E.M., and we used SPSS 10.0 for Windows for analysis of the statistics. The significance level was set at $p<0.05$. 
Table 2. Changes of the Latency Time during $6 \mathrm{~d}$ of the Acquisition Test in the Morris Water Maze Task

\begin{tabular}{|c|c|c|c|c|c|c|c|}
\hline \multirow{2}{*}{ Group } & \multirow{2}{*}{$n$} & \multicolumn{6}{|c|}{ Time (d) } \\
\hline & & 1 & 2 & 3 & 4 & 5 & $6(s)$ \\
\hline Normal & 6 & $132.4 \pm 25.8$ & $67.2 \pm 31.9$ & $40.1 \pm 12.0$ & $24.8 \pm 8.5$ & $27.0 \pm 7.9$ & $27.9 \pm 10.4$ \\
\hline Control & 6 & $122.8 \pm 21.7$ & $107.8 \pm 26.5$ & $50.4 \pm 21.1$ & $29.5 \pm 10.6$ & $23.6 \pm 10.3$ & $26.6 \pm 5.0$ \\
\hline SOY & 6 & $112.2 \pm 26.2$ & $86.1 \pm 22.0$ & $64.7 \pm 17.6$ & $56.7 \pm 10.1$ & $50.4 \pm 8.8$ & $51.7 \pm 3.8$ \\
\hline
\end{tabular}

Each value represents the mean \pm S.E.M.

\section{RESULTS}

Morris Water Maze Test Table 2 shows the mean group latencies to reach the hidden platform in the MWM for all groups for the $6 \mathrm{~d}$. There were no significant main effects, but there was a slight trend for a significant interaction effect on the distance traveled to reach the platform.

On the retention test on the 7 th day, the latency times $(\mathrm{s} / \mathrm{min} \%)$ were $6.2 \pm 1.2,2.7 \pm 0.8$ and $6.3 \pm 2.2$ for the normal, control and SOY groups, respectively $(p<0.05)$. Analysis of the performance on the probe trial for comparing the percentage of time spent swimming around the platform is illustrated in Fig. 1. The time spent around the platform among the groups differed, and the normal and SOY groups spent more time around the platform than did the control group.

Elevated Plus Maze Test ANOVA revealed a significant effect of the differences in the EPM performance between the normal and control groups (Tables 3A, B). For the locomotor activity, the SOY group made significantly more entries into the open arms than did the control group $(p<0.05$, Table $3 \mathrm{~A})$. For the anxiety reaction, the control group remained for $81.6 \pm 3.7 \%$ in the open arms $(p<0.05)$, whereas the SOY group spent more time, i.e., $93.3 \pm 2.9 \%$ in the open arms (Table 3B).

ChAT Immunohistochemistry The mean numbers of ChAT-positive neurons (number $/ 4 \mathrm{~cm}^{2}$ ) in the CA3 and CA1 regions were $18.4 \pm 0.6$ and $16.0 \pm 0.2$ for the normal group, respectively, and $12.9 \pm 0.3$ and $11.1 \pm 0.1$ for the control group, respectively. The ChAT-positive neurons in the controls were significantly reduced compared to the normal group in these regions $(p<0.05)$. However, for the SOY group, the mean numbers of ChAT-positive neurons expressed were $15.8 \pm 1.2$ and $12.6 \pm 0.3$ in each region, respectively. The density of the ChAT-positive neurons of the SOY group in the hippocampus CA1 and CA3 regions were increased significantly compared to the control group (Fig. 2).

AChE Histochemistry The mean numbers of AChEpositive neurons in the $\mathrm{CA} 3$ region were $18.8 \pm 0.3,13.2 \pm 0.4$ and $17.7 \pm 0.3$ for the normal, control and SOY groups, respectively (Fig. 3). The AChE reactive neurons in the controls were significantly reduced compared with the normal group $(p<0.05)$. In the SOY group, a marked increase compared to the control group could be observed $(p<0.05)$.

In the CA1 region (Fig. 3), the AChE-positive neurons (number $/ 4 \mathrm{~cm}^{2}$ ) were $19.6 \pm 0.5,13.4 \pm 0.5$ and $17.9 \pm 0.3$ for the normal, control and the SOY groups, respectively. There was significantly lower AChE reactivity in the controls than in the normal group $(p<0.05)$, while for the SOY group, significantly increased AChE-positive neurons also could be observed compared to the control $(p<0.05)$.

Serum Level of Corticosterone The serum levels of

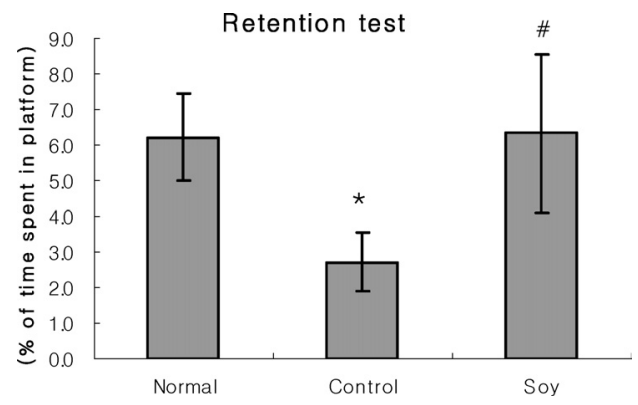

Fig. 1. The Latency Time on the 7th Day of the Retention Test in the Morris Water Maze Task

Comparison of retention performance among the three groups of rats. Separate measures of one-way ANOVA of swimming time among the groups. Each value represents the mean \pm S.E.M. $(n=6) . * p<0.05$ compared to normal and $\# p<0.05$ compared to control.

Table 3A. Number of Entries into the Open and Closed Arms in the Elevated Plus Maze Task

\begin{tabular}{llc}
\hline \hline Group & $n$ & Number $(/ 5 \mathrm{~min})$ \\
\hline Normal & 6 & $15.5 \pm 1.1$ \\
Control & 6 & $10.0 \pm 0.9^{*}$ \\
SOY & 6 & $15.0 \pm 1.4^{\#}$ \\
\hline
\end{tabular}

Each value represents the mean \pm S.E.M. $* p<0.05$ compared to normal and $\# p<0.05$ compared to control.

Table 3B. Time Spent in the Open and Closed Arms in the Elevated Plus Maze Task

\begin{tabular}{llll}
\hline \hline Group & $n$ & Open arms & Closed arms (\%s) \\
\hline Normal & 6 & 100 & 100 \\
Control & 6 & $81.6 \pm 3.7^{*}$ & $116.5 \pm 3.5^{* *}$ \\
SOY & 6 & $93.9 \pm 2.9$ & $106.5 \pm 2.9$ \\
\hline
\end{tabular}

Each value represents the mean \pm S.E.M. $* p<0.05, * * p<0.01$, compared to normal.

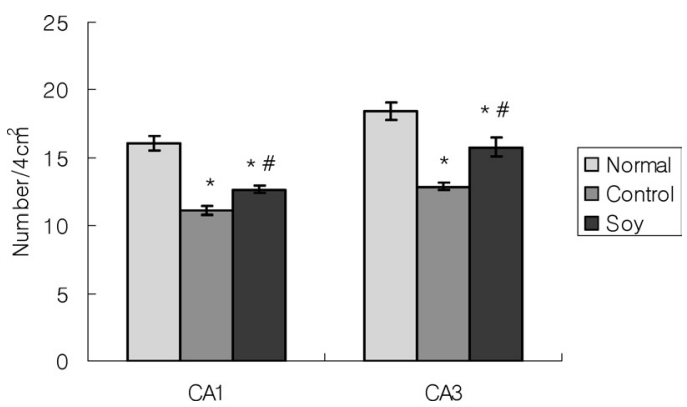

Fig. 2. The Number of ChAT-Immunoreactive Neurons in the Hippocampal CA1 and CA3 of the Experimental Groups

Immunohistochemical data of ChAT were analyzed by performing separate one-way ANOVA of neurons among the groups. Each value represents the mean \pm S.E.M. $* p<0.05$ compared to normal and $\# p<0.05$ compared to control. 


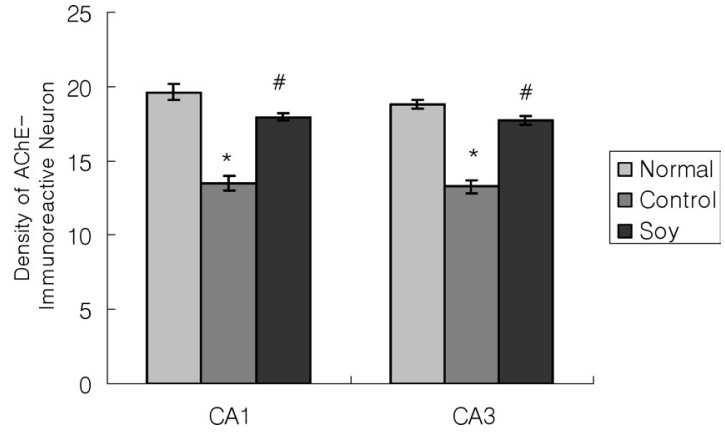

Fig. 3. Density of the AChE-Reactive Neurons in the Hippocampal CA1 and $\mathrm{CA} 3$ of the Experimental Groups

The results of AChE-reactivity were analyzed by performing separate one-way ANOVA of neurons among the groups. Each value represents the mean \pm S.E.M. $* p<0.05$ compared to normal and $\# p<0.05$ compared to control.

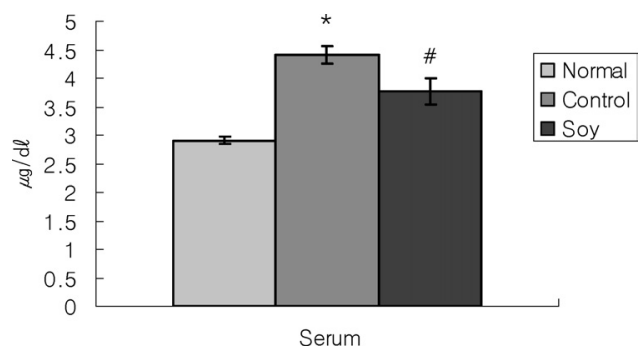

Fig. 4. The Level of Corticosterone of the Experimental Groups

Each value represents the mean \pm S.E.M. $* p<0.05$ compared to normal and $\# p<0.05$ compared to control.

corticosterone were $2.9 \pm 0.1,4.4 \pm 0.2$ and $3.8 \pm 0.2(\mu \mathrm{g} / \mathrm{dl})$ in the normal, control and SOY groups, respectively (Fig. 4). The serum level of corticosterone in the SOY group was significantly lower than that in the control group $(p<0.05)$, while the control group had significantly higher corticosterone levels compared to the normal group $(p<0.05)$.

\section{DISCUSSION}

In the present study, treatment with Soyo-san improved the function of memory retention in the MWM; it also displayed an anxiolytic-like effect in the EPM and increased ChAT immunoreactivity and $\mathrm{AChE}$ reactivity in the hippocampal CA3 and CA1 areas of the ovariectomized and immobilizationstressed female rats. In addition, we observed reduced corticosterone levels in the Soyo-san treated group compared to the control group.

Female animals show a higher magnitude and duration of the corticosterone response after both neurogenic and systemic stressors. ${ }^{20)}$ At the biological level, stress-related psychopathologies share profound alterations of the HPA axis and sex-differences in the HPA function have been proposed to contribute to the greater vulnerability of women to stress. $^{21,22)}$ Very low levels of estradiol (as seen during menopause or in ovariectomized (OVX) female rodents) or very high levels (as seen during the mid-luteal phase or during proestrus) are associated with impaired spatial learning ability. ${ }^{23-25)}$ Sex differences in performance on behavioral tests may be mediated by the physiological correlates of the variations in the endocrine status. The estrous cycle is associated with the basal and stress-related levels of glucocorti- coids and ACTH. The hormonal systems of the HPA axis may influence performance in the water maze task. ${ }^{26,27)}$ Both spatial memory and HPA responsiveness to stress are partly controlled by the hippocampus. ${ }^{28)}$ Studies on rodents and people indicate that stress or glucocorticoid administration can impair hippocampus-specific learning and memory. ${ }^{29,30)}$ Another interpretation of these studies may be that the sexrelated and cycle-related variations are due to differential responses to stress when the tasks are being performed.

Soyo-san has been used to effectively manage stress and depression-related disorders in Korea. In laboratory studies of animals, Soyo-san has also been shown to have antidepressant-like effects using the tail suspension and forced swimming tests. ${ }^{15,16)}$ Bupleuri Radix is one of the most important constituents of Soyo-san, and it caused a significant anti-immobility effect on the FST and reversed the CMS-induced reduction of sucrose consumption. ${ }^{31)}$ Several Japanese studies have suggested that Saiko-ka-ryukotsu-borei-to, an herbal compound containing Bupleuri Radix, has antidepressant properties. This herbal medicine ameliorated stress-induced immobility and the associated biochemical changes. ${ }^{32,33)}$ Essential oil of Angelicae Gigantis Radix, a component of Soyo-san, exhibited an anxiolytic-like effect in the elevated plus-maze, the light/dark box and the stress-induced hyperthermia paradigms in mice. ${ }^{34)}$ Matsumoto et al. reported that ligustilide, an ingredient of Angelicae Gigantis Radix, had a beneficial effect on psychological stress-induced pathophysiological changes in the central nervous system. For example, it could reverse the decrease in the duration of pentobarbital sleep in mice that was caused by social isolation stress and activation of the central noradrenergic system. ${ }^{35)}$ Toriizuka et al. reported that Menthae Herba, a component of Soyosan, increased the social interaction behavior in mice, indicating an anxiolytic effect. ${ }^{36}$ Paeoliae Radix Alba is reported to influence the processes related to the mechanism of antidepressant action. ${ }^{37)}$ These findings show that Soyo-san acts on the central nervous system. The present study found that Soyo-san treatment in ovariectomized and immobilizationstressed female rats prevented forgetting during retention of the MWM task. The results confirm the ability of Soyo-san to prevent ovariectomized female rats from displaying forgetfulness in this task. The consequences of exposure to anxiety upon stress were measured using the EPM test, which is probably the most popular animal model of anxiety. The EPM is widely used to study rodent anxiety, and the classical indices of anxiety-related behavior are the number of open arm entries and the time spent on the open arms. An anxiolytic agent increased the frequency of entries into open arms and increased the time spent in the open arms of the EPM. ${ }^{38)}$ Anti-anxiety drugs and SSRIs (selective serotonin reuptake inhibitors) have a reduction effect on anxiety reaction in an elevated plus maze. ${ }^{18)}$ In the present study, Soyosan treatment prolonged the time spent in the open arms and the number of entries into the open arms compared to the control group. For the anxiety reaction, the stressed animal spent a longer time in the closed arms did the normal group.

Our results showed that Soyo-san treatment significantly increased the ChAT and AChE expression. ChAT and AChE belong to a family of enzymatic proteins that are distributed in cholinergic neurons. ChAT is the biosynthetic enzyme of $\mathrm{ACh}$ and is a key marker that is required for cholinergic neu- 
rotransmission in the central and peripheral nervous systems. In particular, $\mathrm{ACh}$ is known to be rapidly hydrolyzed by AChE. The duration of action of ACh at the synaptic clefts is critically dependent on AChE activity. ${ }^{39)}$ Cholinergic neurons originating in the nucleus basalis of Meynert and the medial septum project to areas such as the cortex and hippocampus, which play a role of ACh in cognition. ${ }^{40,41)}$ The hippocampus has been associated with memory processes and it is densely innervated by cholinergic fibers. ${ }^{42)}$ Gottesfeld et al. showed a decrease in ChAT activity after repeated immobilization stress. $^{43)}$ After electroshock, the ACh concentration was found to be reduced in the brain of rats. ${ }^{44)}$ Mitsushima et al. reported that circulating estrogen could directly activate cholinergic neurons to sustain the ACh stress response in the hippocampus of female rats. ${ }^{45)}$ The estrous cycle is associated with basal and stress-related levels of glucocorticoids and ACTH. ${ }^{24)}$ Soyo-san treatment significantly decreased the serum level of corticosterone that had been elevated by stress. Several groups have shown that chronic exposure to stress or exogenous corticosterone leads to neuronal remodeling in the key brain regions associated with depressive illness, namely the hippocampus. ${ }^{46}$

Ovarian failure and termination of reproductive female functions can influence the mental and the cognitive activity of menopausal women. Although the mechanisms underlying menopausal mental disorders are unclear, the decline in ovarian steroids produces functional changes in the central nervous system. ${ }^{2,47)}$ Clinical studies have indicated that estrogen replacement improves mood and it prevented some disorders in post-menopausal women who had no or mild depressive symptoms. ${ }^{48,49)}$ However, in postmenopausal women with major depression, estrogen alone is not sufficient, although it may be useful as an augmentation strategy. Thus, it is important to investigate this potential remedy for treating the major depression induced by menopause. We suggest that Soyo-san can be useful for treating menopausal depression in patients. Further studies are necessary to improve our understanding of the pharmacology of Soyo-san and to elucidate the mechanism of action of these effects using Soyo-san's isolated active components and purified ingredients for the treatment of depression.

Acknowledgments This work was supported by the Neurobiology Research Program of the Korea Ministry of Science and Technology, Korea 2004-01757.

\section{REFERENCES}

1) Weissman M. M., Livingston B. M., Leaf P. J., Florio L. P., Holzer C. I., "Affective Disorders," ed. by Robins L. E., Regier D. A., The Free Press, New York, 1991, pp. 53-80.

2) Shors T. J., Leuner B., J. Affective Disorders, 74, 85-96 (2003).

3) Desmond N. L., Levy W. B., Hippocampus, 7, 239-245 (1997).

4) Smith C. C., Cizza G., Gomez M., Greibler C., Gold P. W., Sternberg E. M., Neuroimmunomodulation, 1, 231-235 (1994).

5) Woolley C. S., Curr. Opin. Neurobiol., 9, 349-354 (1999).

6) Kellendonk C., Gass P., Kretz O., Schutz G., Tronche F., Brain Res. Bull., 57, 73-83 (2002).

7) McEwen B. S., Ann. N.Y. Acad. Sci., 746, 134-142 (1994).

8) Sapolsky R. M., Biol. Psychiatry, 48, 755-765 (2000).

9) Sheline Y. I., Biol. Psychiatry, 48, 791-800 (2000).

10) Bremner J. D., Naraya M., Anderson E. R., Staib L. H., Am. J. Psychiatry, 157, 115-117 (2000).
11) Janowsky D. S., Overstreet D. H., "Psychopharmacology, The Fourth Generation of Progress," ed. by Bloom F. E., Kupfer D. J., Raven Press, New York, 1995, pp. 945-956.

12) Fibiger H. C., Damsma G., Day J. C., "The Basal Forebrain," ed. by Napier T. C., Kalivas P. W., Hanin I., Plenum Press, New York, 1991, pp. $399-414$

13) Fan D. H., Chin. Tradit. Herb. Drugs, 18, 44-45 (1996).

14) Zhao L. J., Chin. J. Hosp. Pharm., 3, 178-179 (2003).

15) Wang J. Y., Shi Y. H., Cha P. Z., Li F. Y., Chin. J. Hosp. Pharm., 22 489-491 (2002).

16) Xu Z. W., Wu L. L., Yan C., Wang W. Z., Ao H. Q., Tradit. Chin. Med Res., 16, 14-15 (2003).

17) Seligman M. E., J. Abnorm. Psychol., 87, 165-179 (1997).

18) Pellow S., Chopin P., File S. E., Briley M., J. Neurosci. Methods, 14, 149-167 (1985).

19) Paxinos G., Watson C., "The Rat Brain in Stereotaxic Coordinates," Academic, San Diego, 1986.

20) Weinstock M., Razin M., Schorer-Apelbaum D., Men D., McCarty R., Int. J. Dev. Neurosci., 16, 289-295 (1998).

21) Ehlert U., Gaab J., Heinrichs M., Biol. Psychol., 57, 141-152 (2001).

22) Young E. A., J. Gend. Specif. Med., 1, 21-27 (1998).

23) Frye C., Physiol. Behav., 57, 5-14 (1995).

24) Galea L. A., Kavaliers M., Ossenkopp K. P., Hampson E., Horm. Behav., 29, 106-125 (1995).

25) Hogervorst E., Williams J., Budge M., Riedel W., Jolles J., Neuroscience, 101, 485-512 (2000).

26) Viau V., Meaney M. J., Endocrinology, 129, 2503-2511 (1991).

27) Oitzl M. S., deKloet E. R., Behav. Neurosci., 106, 62-71 (1992).

28) Jacobson L., Sapolsk R., Endocr. Rev., 12, 118-164 (1991).

29) Diamond D. M., Park C. R., Heman K. L., Rose G. M., Hippocampus, 9, 542-552 (1999).

30) Wolf O. T., Schommer N. C., Hellhammer D. H., McEwen B. S., Kirschbaum C., Psychoneuroendocrinology, 26, 711-720 (2001).

31) Kim S. H., Han J., Seog D. H., Chung J. Y., Kim N., HongPark Y., Lee S. K., Life Sci., 76, 1297-1306 (2005).

32) Mizoguchi K., Yuzurihara M., Ishige A., Aburade M., Tabira T., Pharmacol. Biochem. Behav., 75, 419-425 (2003).

33) Mizoguchi K., Yuzurihara M., Ishige A., Sasaki H., Tabira T., Life Sci., 72, 67-77 (2003).

34) Chen S. W., Min L., Li W. J., Kong W. X., Li J. F., Zhang Y. J., Pharmacol. Biochem. Behav., 79, 377-382 (2004).

35) Matsumoto K., Kohno S., Ojima K., Tezuka Y., Kadota S., Watanabe H., Life Sci., 62, 2073-2082 (1998).

36) Toriizuka K., Kamiki H., Ohmura N. Y., Fujii M., Hori Y., Fukumura M., Hirai Y., Isoda S., Nemoto Y., Ida Y., Life Sci., 77, 3010-3020 (2005).

37) Liu T. P., Liu M., Tsai C. C., Lai T. Y., Hsu F. L., Cheng J. T., J. Pharm. Pharmacol., 54, 681-688 (2002).

38) Rodgers R. J., Dalvi A., Neurosci. Biobehav. Rev., 21, $801-810$ (1997).

39) Cooper J. R., Bloom F. E., Roth R. H., "Acetylcholine, The Biochemical Basis of Neuropharmacology," Oxford University Press, New York, 2003, pp. 151-170.

40) Satoh K., Armstrong D. M., Fibiger H. C., Brain Res. Bull., 11, 693720 (1983).

41) Mckinney M., Coyle J. T., Hedreen J. C., J. Comp. Neurol., 217, 103121 (1983).

42) Huang Y. L., Onodera H., Takeda A., Itoyama Y., Kogure K., Brain Res., 722, 195-199 (1996).

43) Gottesfeld Z., Kvetnansky R., Kopin I. J., Jacobowitz D. M., Brain Res., 152, 374-378 (1978).

44) Longoni R., Mulas A., Novak B. O., Pepeu I. M., Pepeu G., Neuropharmacology, 15, 283-286 (1976).

45) Mitsushima D., Masuda J., Kimura F., Neuroendocrinology, 78, 234 240 (2003).

46) Woolley C. S., Gould E., McEwen B. S., Brain Res., 531, 225-231 (1990).

47) Pearlstein T., Rosen K., Stone A. B., Endocrinol. Metab. Clin. North Am., 26, 279-294 (1997).

48) Rubinow D. R., Schmidt P. J., Roca C. A., Biol. Psychiatry, 44, 839850 (1998).

49) Burt V. K., Altshuler L. L., Rasgon N., Harv. Rev. Psychiatry, 6, 121 132 (1998). 